LAWRENCE LIVERMORE NAT IO N A L LABORATORY

\title{
Molecular Clouds: Observation to Experiment
}

J. O. Kane, D. D. Ryutov, A. Mizuta, B. A.

Remington, M. W. Pound

May 12, 2004

HEDLA 2004

Tucson, AZ, United States

March 10, 2004 through March 13, 2004 
This document was prepared as an account of work sponsored by an agency of the United States Government. Neither the United States Government nor the University of California nor any of their employees, makes any warranty, express or implied, or assumes any legal liability or responsibility for the accuracy, completeness, or usefulness of any information, apparatus, product, or process disclosed, or represents that its use would not infringe privately owned rights. Reference herein to any specific commercial product, process, or service by trade name, trademark, manufacturer, or otherwise, does not necessarily constitute or imply its endorsement, recommendation, or favoring by the United States Government or the University of California. The views and opinions of authors expressed herein do not necessarily state or reflect those of the United States Government or the University of California, and shall not be used for advertising or product endorsement purposes. 
For submittal to Proceedings of HEDLA 2004, in Astrophysics and Space Science Journal.

\title{
MOLECULAR CLOUDS: OBSERVATION TO EXPERIMENT
}

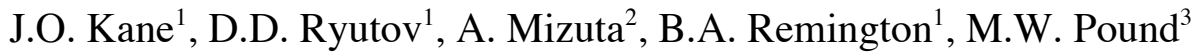 \\ ${ }^{1}$ Lawrence Livermore National Laboratory, Livermore, CA 94551, USA \\ ${ }^{2}$ Institute of Laser Engineering, Osaka University, Osaka, 565-0871, Japan \\ ${ }^{3}$ Astronomy Department, University of Maryland, College Park, MD 20742, USA
}

\begin{abstract}
Our ongoing investigation of how 'Pillars' and other structure form in molecular clouds irradiated by ultraviolet (UV) stars has revealed that the Rayleigh-Taylor instability is strongly suppressed by recombination in the photoevaporated outflow, that clumps and filaments may be key, that the evolution of structure is well-modeled by compressible hydrodynamics, and that directionality of the UV radiation may have significant effects. We discuss a generic, flexible set of laboratory experiments that can address these issues.
\end{abstract}

\section{Introduction}

The Eagle Nebula is a molecular cloud irradiated by ultraviolet (UV) stars, and displays remarkable evolving structure, in particular its famous 'Pillars' and smaller 'EGGS' (Hester; 1996; Pound, 1998). Our ongoing investigation of the formation of such structures (Ryutov et al., 2004) involves a combination of theory, computer simulations, and laboratory experiments that mock up key aspects of the relevant physics. Our investigation to date suggests clear directions for such experiments.

Among the results of our work to date are the following: (1) recombination of ionized $\mathrm{H}$ in the tenuous outflowing photoevaporated (ablated) material suppresses Rayleigh Taylor growth (A. Mizuta, this issue); this suggests a key role for the pre-existing clumpy, filamentary structure typical of molecular clouds. (2) Direct drive laser experiments at currently available energies allow experiments that address the clump/filament model, and also address observations of the Pillars. (3) Some structure formation may be due to effects of the directionality of the UV radiation - the tilted radiation instability (TR - D. Rytuov, this issue) and general directed radiation (DR) effects. (4) Experiments at higher energy lasers or pulsed-power machines may allow us to study such directionality effects, using a 'flashlight' drive from a radiation cavity (hohlraum). Experiments using direct laser illumination (direct drive) may allow directionality experiments at currently available facilities, if the distance between the absorption (critical) surface and the ablation front remains small enough to preserve the directional character of the incoming radiation.

\section{Generic, flexible set of laser experiments}

We can select from a generic, flexible set of laser experiments to address critical issues in the formation of structures in molecular clouds. Fig. 1 shows a generic target and two choices for drive - direct laser illumination and indirect illumination via a hohlraum. In experiments, we represent the molecular cloud by a uniform background material such as $\mathrm{C}$ foam, within which we embed rods ('filaments') or spheres ('clumps') of a denser material 
such as C (graphite). Initial surface (facing the drive) or other perturbations can be added to trigger Rayleigh-Taylor or Richtmyer-Meshkov hydrodynamic instabilities. Varying the density contrast between the cloud and the embedded material can be used to produce differential dynamic response of the target, in particular to shocks. At laser facilities, compared to indirect drive, direct drive generally allows a simpler target, a longer pulse (permitting more evolution of structure), and higher total energy delivered to the target, which may permit testing aspects of the experiments at smaller lasers.

The surface of the target can be tilted with respect to the average normal direction of the drive, allowing investigation of TR and DR. Directionality experiments may be possible with direct drive if the standoff distance $L_{\mathrm{c}}$ (the distance from the critical surface to the ablation front, between which the drive energy is transported by electron conduction) remains small enough that the ablation front still sees directional effects. Finally, a hohlraum flashlight drive possible at a higher energy facility may allow greater directionality without the complicating issue of the standoff distance.
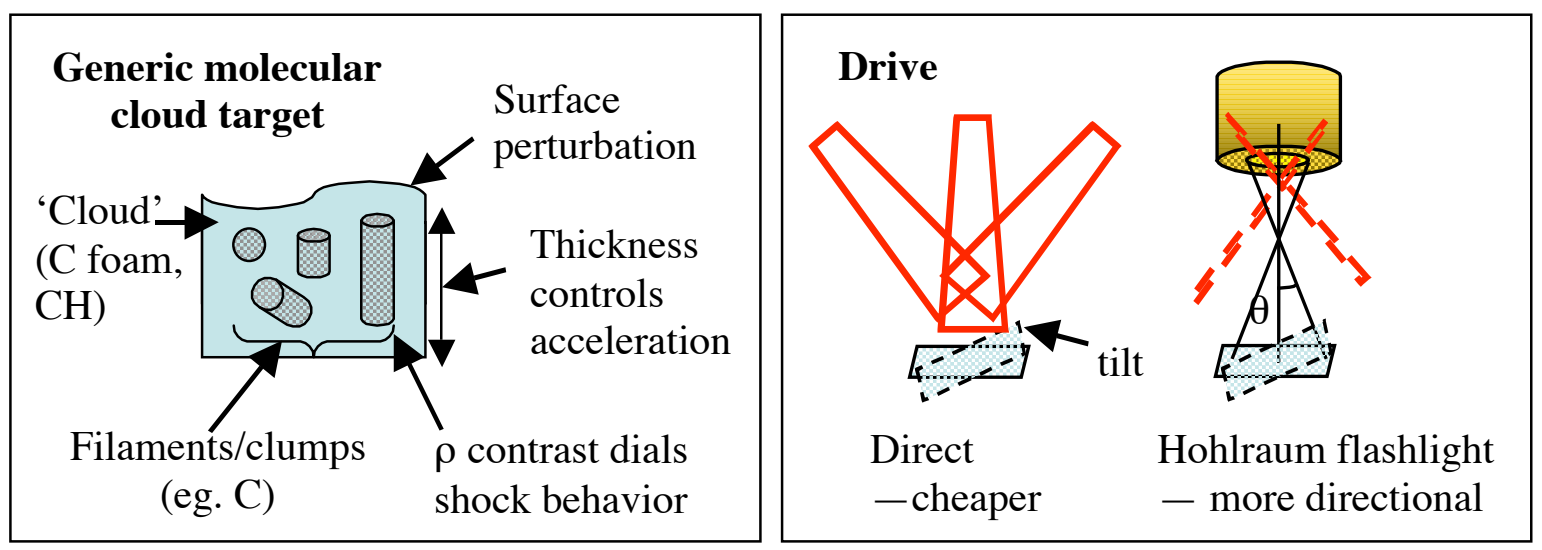

Figure 1.

As illustrated in Fig. 2, preliminary simulations and other design work suggests a set of five experiments. A single embedded filament or clump may produce a final structure with a number of diagnosable features. In particular, a filament may produce a structure similar in appearance to a Pillar. Such experiments allow a two-step comparison of embedded filament/clump models to detailed velocity and density data for the Pillars of the Eagle Nebula (Pound, 1998). First, we confirm that simulations reproduce diagnosable features. Second, having validated our simulations, we then compare the detailed density and velocity information in these simulations to the astrophysical data. In this way, we may evaluate Rayleigh-Taylor and other models of structure formation with controlled experiments. With higher energy facilities, we may be able to drive and diagnose larger targets with multiple embedded structures, allowing us to investigate the complicated response of a more realistically structured cloud.

Experimental techniques suitable for TR exist (Azechi et al., 1997). Meanwhile, simulations by our group and others (Williams, 2002) have shown what we call the directed radiation (DR) instabilities, short wavelength instability of an ablating surface occurring in the absence of acceleration or a shock. It appears that DR may seed extensive structure formation due to shadowing effects, and may be mitigated by recombination. Theoretical and numerical work have yet to show whether DR has significant physical effects or should 


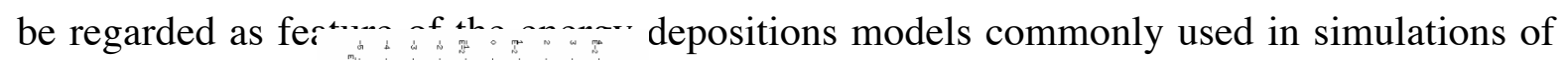
molecular clouds.

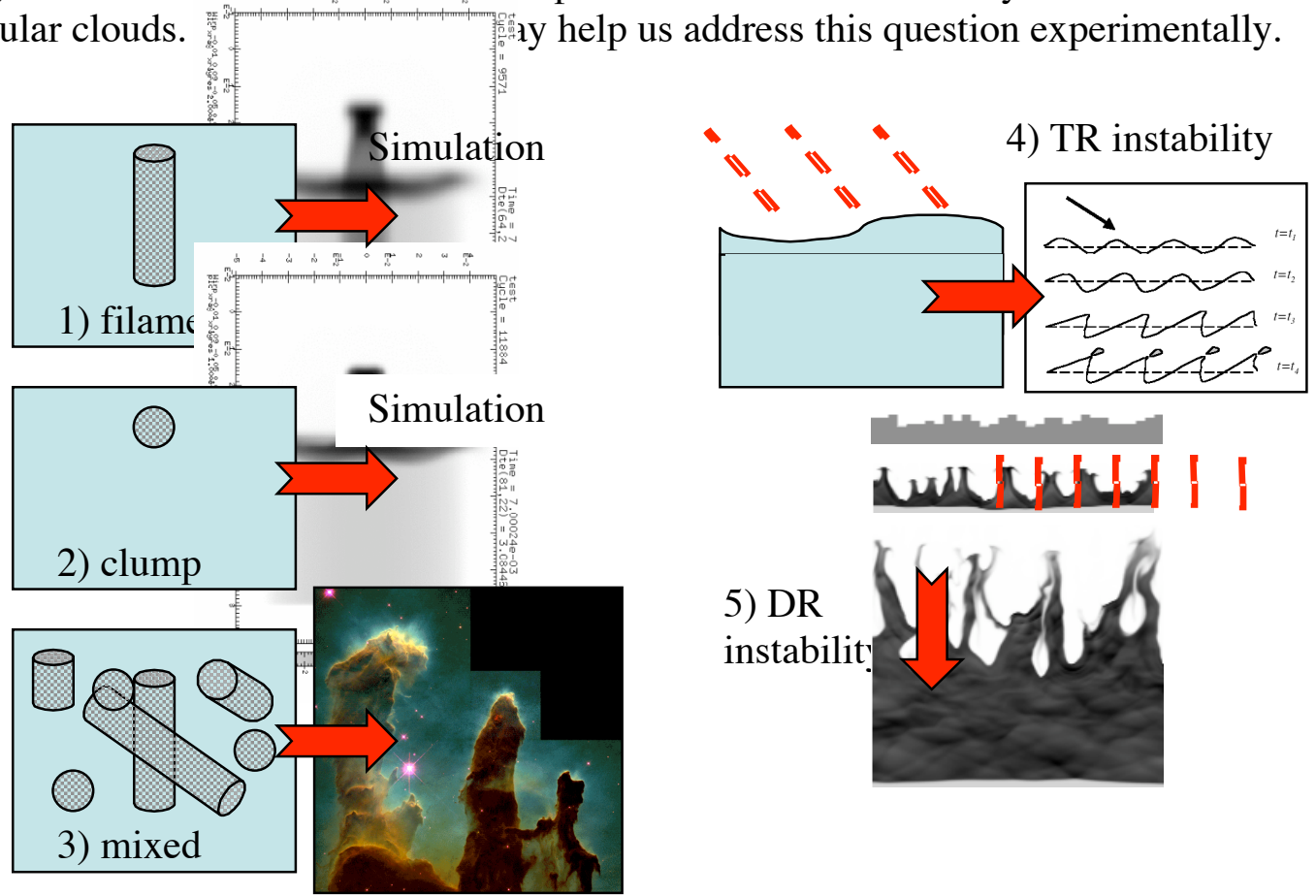

Figure 2 .

\section{Design of a Clump/filament Experiment}
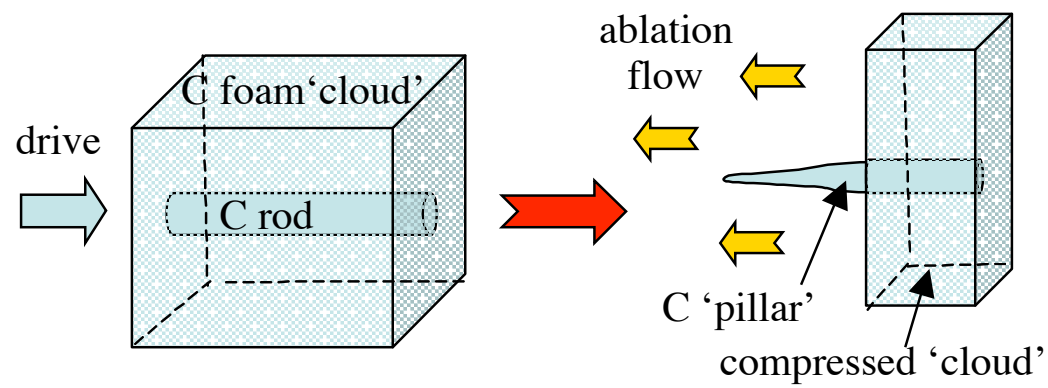

Figure 3.

We have designed a first experiment in which we embed a $\mathrm{C}$ rod in $\mathrm{C}$ foam parallel to the drive (Fig. 3). Our goal is to produce a shocked, ablating pillar-like structure as the lighter foam is compressed past the rod. We wish to produce several diagnosable features we can use to validate our hydrodynamic simulations of the experiments, then compare the detailed velocity and density profiles in the simulations to observations of Column II of the Eagle Nebula (Pound, 1998.) As a first step, we simulate the $\mathrm{C}$ rod-C foam experiment using the LLNL hydrodyamics code CALE. We simulate a $200 \mu \mathrm{m}$ diameter $\mathrm{C}$ rod embedded in a block of $0.25 \mathrm{~g} / \mathrm{cm}^{3} \mathrm{C}$ foam. We approximate the drive with a single beam at normal incidence, at laser wavelength $\lambda_{\mathrm{L}}=0.35 \mu \mathrm{m}$. The drive pulse is $2100 \mathrm{~J}$ in a $7 \mathrm{~ns}$ square pulse. We assume half the energy is absorbed at the surface of critical electron density $\rho_{\mathrm{e}}\left(\lambda_{\mathrm{L}}\right)$, the rest reflected out of the problem. The laser spot size has intensity profile $\mathrm{I} \sim \exp [-$ $\left.(\mathrm{r} / 411 \mu \mathrm{m})^{4.6}\right]$. We assume a backlighter in the 5-6 keV range will be used to produce sideon radiographs; because directly-driven carbon may self-emit in this range, we use 
multigroup radiation transport including a $(5,6) \mathrm{keV}$ bin. Assuming that to mitigate selfemission we may want to wait until after the drive ends before taking data, we examine how self-emission drops after the drive ends, and whether there is significant attendant hydrodynamic expansion of the target. For simulated radiographs, we assume a diagnostic blurring characterized by a $20 \mu \mathrm{m}$ Gaussian.

The results are shown in Fig. 4. The conclusions are as follows. (1) Radiograph quality and features: the ablation front and the shock are observable in both the $\mathrm{C}$ and the $\mathrm{C}$ foam, allowing validation of simulations. (2) Self-emission drops rapidly after drive ends. (3) Hydrodynamic expansion is insignificant for 300 ps after drive ends, so self-emission can be mitigated if needed simply by waiting.
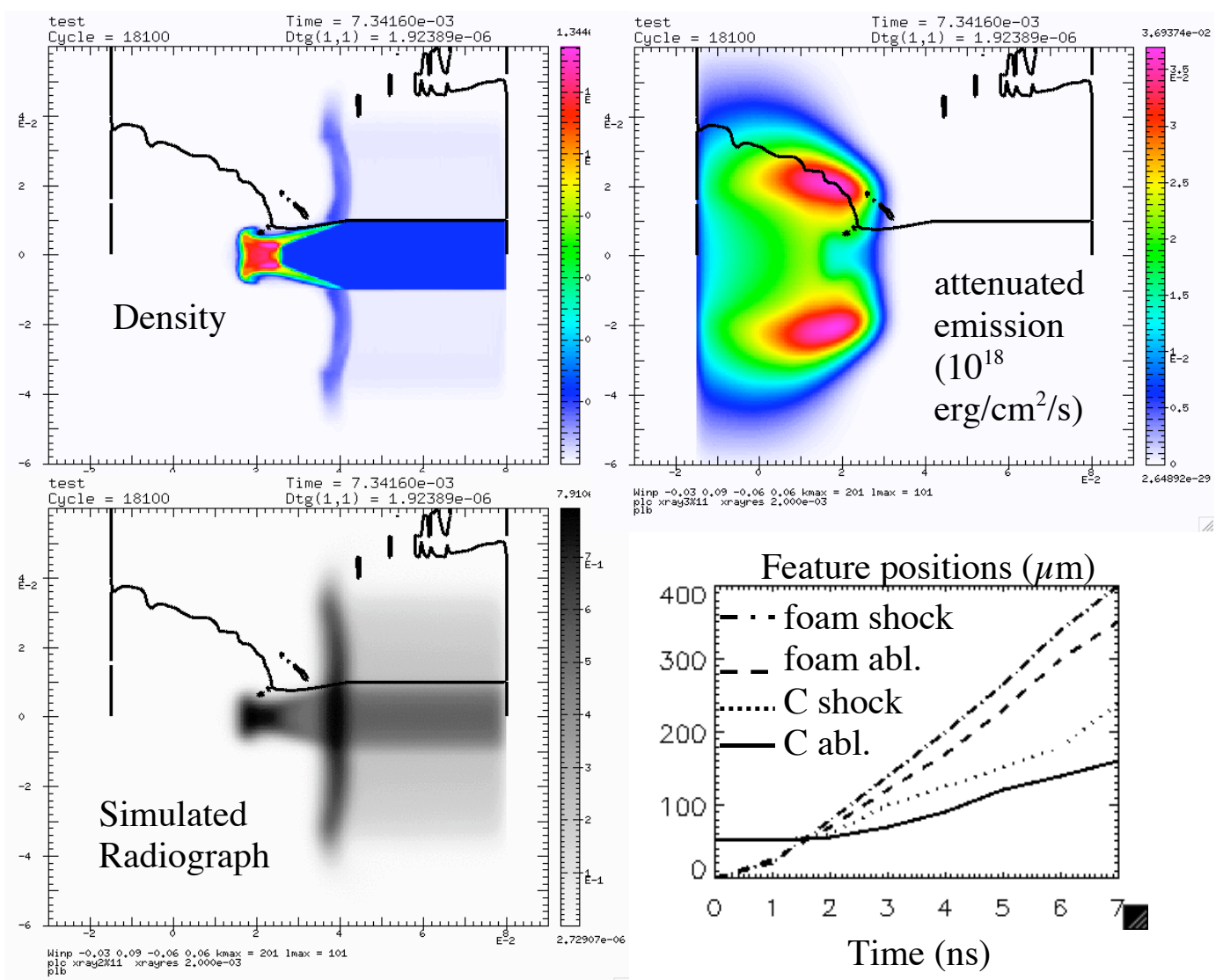

Figure 4.

\section{References}

Proc. of the Intern. Conf. held at Estes Park, Colorado, May 19-23 1997, v.148, C.E.

Woodward, J.M. Shull, H.A. Thronson, Jr. - Eds., p150.

Azechi et al., Phys. Plasmas 4 (11), November 1997

Hester, J.J., Scowen, P.A., Sankrit, R. et al: 1996, Astronomical Journ. 1112349.

Levenson, N., Graham, J.R., McLean, I.S., et al: 2000, ApJ, 533, L53.

Mizuta, A et al., this issue

Pound, M.W.: 1998, ApJ, 493, L113.

Pound, M.W. et al., this issue.

Ryutov, D.D., Remington, B.A.: 2002, Plasma Phys. Contr. Fus., 44, B407. 
Ryutov, D.D., Kane, J.O., Mizuta, A., et al.: 2004. In: “Plasmas in the Laboratory and in the Universe”, AIP Conference Proceedings, Volume 703, p. 415, Melville, NY.

Ryutov, D.D. et al., this issue

Williams, R. J. R., Mon. Not. R. Astron. Soc. 331, 693-706 (2002).

This work was performed under the auspices of the U.S. Department of Energy by the University of California, Lawrence Livermore National Laboratory under Contract No. W-7405-Eng-48. 